\title{
Study of Prevalence of Diabetic Retinopathy in Already Confirmed Diabetic Patients Who Were Reffered for Screening Purpose
}

\author{
Authors \\ Parvez Ahmad Bhat ${ }^{1}$, Jasia Ishtiaq ${ }^{2}$, Arsalan un Nisa ${ }^{3}$, Wallied K Ballwan ${ }^{4}$, \\ Wani Prince Muzaffar ${ }^{5}$ \\ ${ }^{1,2}$ Senior Resident, Department of Ophthalmology, SKIMS Srinagar \\ ${ }^{3}$ Postgraduate Scholar, Department of Ophthalmology, GMC Srinagar \\ ${ }^{4}$ Postgraduate Scholar, Department of Community Medicine, SKIMS Srinagar \\ ${ }^{5}$ Senior Resident, Department of General Surgery, SKIMS Srinagar \\ Correspondence Authors \\ Dr. Parvez Ahmad Bhat \\ Senior Resident, Department of Ophthalmology, SKIMS Srinagar (J\&K) India \\ Email: bhatparvez44@gmail.com, Cell: +91-9906483578
}

\section{ABSTRACT}

Objective: To study prevalence of diabetic retinopathy among diabetic patients referred for screening purpose.

Methods: Total of 2716 diabetic patients who were referred to our ophthalmology department for screening were included in the study. Complete eye examination including visual acuity, anterior segment examination and dilated fundus exam was performed. Age specific prevalence of diabetic retinopathy was recorded and also duration of diabetes was considered. Diabetic retinopathy if present was graded according to ETDRS grading system.

Results: Out of 2716 patients in 2273 (84\%) revealed no diabetic retinopathy and in 443 (16\%) revealed varying degree of diabetic retinopathy. Most of the diabetic retinopathy patients were aged between 60-90 years and least number were between 37-60 years age group. Duration of diabetes was the single most important risk factor for diabetic retinopathy. 56\% of diabetic retinopathy patients had duration of diabetes of more than 10 years, where 12\% had 5-10 years duration and only 3\% had duration of diabetes of less than 5 years. Mild NPDR was the most common type of diabetic retinopathy (52\%) followed by severe NPDR (22\%), moderate NPDR (17\%), NPDR with CSME (6\%) and PDR (3\%).

Conclusion: Diabetic retinopathy is a visually blinding complication of diabetes. Two most important ways to prevent it is to exercise a strict glycemic control and to do a regular dilated fundus examination to detect it at an early stage.

Keywords: NPDR (non-proliferative diabetic retinopathy), PDR (proliferative diabetic retinopathy), CSME (clinically significant macular edema), ETDRS (Early treatment diabetic retinopathy study). 


\section{INTRODUCTION}

Diabetic retinopathy (DR) is the leading cause of visual impairment in the western world, particularly among persons of working age group. ${ }^{1}$ It is estimated that diabetic retinopathy develops in more than $75 \%$ of diabetic patients within 15 to 20 years of diagnosis of diabetes. ${ }^{2}$ Several epidemiological studies have provided valuable information on the prevalence of DR in western countries that is useful for identifying subgroups at risk and for planning public health policies. ${ }^{3}$ However there is a paucity of data on the prevalence of diabetes related ocular diseases in developing countries especially India, which in fact has the largest number of diabetes patients in the world.

According to the WHO report India has 31.7 million diabetic subjects, and the number is expected to increase to 79.4 million by $2030 .^{4}$ Furthermore type- 2 diabetes in Indians differs from that of Europeans in several aspects like onset at younger age ${ }^{5}$ and genetic factors appear to be more stronger. The clinical differences and rising prevalence of diabetes in India ${ }^{6}$ warrant well conducted epidemiological studies on diabetes related complications in this population to assess the health services burden due to diabetes.

\section{MATERIAL AND METHODS}

A total of 2716 patients who were already diagnosed with diabetes mellitus and were referred to department of ophthalmology SKIMS Srinagar J\&K, India for screening for diabetic retinopathy were included in the study. Ethics committee approval was sought and informed consent was obtained from all patients. A detailed history was taken including age, sex, time of diagnosis, duration of diabetes, drug intake history, family history and any associated systemic illness.

Complete eye examination was performed by skilled ophthalmologists including visual acuity by Snellen acuity chart, anterior segment examination by slit lamp, detailed fundus examination by direct ophthalmoscopy and indirect ophthalmoscopy using 20D lens. Diabetic retinopathy if present was graded according to ETDRS grading system into non-proliferative diabetic retinopathy (NPDR), which was further divided into mild, moderate and severe and proliferative diabetic retinopathy (PDR). Patients diagnosed with severe NPDR, PDR or clinically significant macular edema (CSME) were referred to tertiary care hospital for further management.

\section{RESULTS}

In our study a total of 2716 patients of already diagnosed diabetes were enrolled, out of which 1435 were males and 1281 were females. Our patients ranged between 37 to 91 years (average 61 years). Out of 2716 patients, in 2273 no diabetic retinopathy changes were detected on fundus examination, while fundus examination revealed varying degree of diabetic retinopathy in 443 patients. Table-1 shows age specific prevalence of diabetic retinopathy in various age groups.

Table-1: Age wise prevalence of diabetic retinopathy.

\begin{tabular}{|c|c|c|c|}
\hline Age (years) & No Retinopathy No. $(\boldsymbol{\%})$ & With Retinopathy No. $(\boldsymbol{\%})$ & Total \\
\hline $37-46$ & $335(92 \%)$ & $30(8 \%)$ & 365 \\
\hline $47-56$ & $365(89 \%)$ & $45(11 \%)$ & 410 \\
\hline $57-66$ & $651(88 \%)$ & $89(12 \%)$ & 740 \\
\hline $67-76$ & $614(75 \%)$ & $205(25 \%)$ & 818 \\
\hline $77-86$ & $241(78 \%)$ & $68(22 \%)$ & 309 \\
\hline $87-91$ & $67(92 \%)$ & $6(8 \%)$ & 73 \\
\hline Total & $\mathbf{2 2 7 3}$ & $\mathbf{4 4 3}$ & $\mathbf{2 7 1 6}$ \\
\hline
\end{tabular}




\section{JMSCR Vol.||03||Issue ||08||Page 6928-6932||August}

It was seen that maximum number of patients with diabetic retinopathy fall between 60 to 90 years of age and least number were between 37 t0 59 years of age which may be attributed to longer duration of diabetes in these patients. Duration of diabetes was also recorded in history of patients. Table-2 shows prevalence of diabetic retinopathy according to duration of diabetes.

Table 2: Duration of diabetes and prevelance of diabetic retinopathy

\begin{tabular}{|c|c|c|c|}
\hline $\begin{array}{c}\text { Duration of } \\
\text { DM (years) }\end{array}$ & Total & $\begin{array}{c}\text { Without Diabetic } \\
\text { Retinopathy }\end{array}$ & $\begin{array}{c}\text { With Diabetic } \\
\text { Retinopathy }\end{array}$ \\
\hline$<5$ years & 822 & $805(98 \%)$ & $17(2 \%)$ \\
\hline $5-10$ & 719 & $631(88 \%)$ & $88(12 \%)$ \\
\hline $10-20$ & 539 & $425(79 \%)$ & $114(21 \%)$ \\
\hline$>20$ & 636 & $412(65 \%)$ & $224(35 \%)$ \\
\hline Total & $\mathbf{2 7 1 6}$ & $\mathbf{2 2 7 3}$ & $\mathbf{4 4 3}$ \\
\hline
\end{tabular}

It was seen that maximum number of patients (76\%) with diabetic retinopathy had duration of diabetes of more than 10 years, $20 \%$ had duration of 5-10 years only $4 \%$ had duration of diabetes of less than 5 years. Diabetic retinopathy was graded according to ETDRS grading system into NPDR (mild, moderate, severe, NPDR with or without CSME and PDR). Table-3 Shows grade wise prevalence of diabetic retinopathy.

Table 3: Grade wise diabetic retinopathy seen.

\begin{tabular}{|l|c|}
\hline Type of Diabetic Retinopathy & No (\%) \\
\hline Mild NPDR & $230(52 \%)$ \\
\hline Moderate NPDR & $75(17 \%)$ \\
\hline Severe NPDR & $98(22 \%)$ \\
\hline NPDR with CSMO & $27(6 \%)$ \\
\hline PDR & $13(3 \%)$ \\
\hline Total & $\mathbf{4 4 3}$ \\
\hline
\end{tabular}

It was seen that 230 (52\%) patients had mild NPDR, 75 (17\%) had moderate NPDR, 98 (22\%) had severe NPDR, 27 (6\%) had NPDR with CSME, and only 13 (3\%) had PDR.

\section{DISCUSSION}

Diabetic retinopathy (DR) is one of the few ophthalmic diseases that have defined preventive measures to delay progression of disease and subsequent visual loss. The estimated high prevalence of diabetes by $2025^{7}$ is a matter of concern considering the potential for visual loss associated with diabetic retinopathy. The lack of fundus photographs is limitation of our study because it is possible that we may have missed some early diabetic retinopathies and underestimated the prevalence of diabetic retinopathy. The strength of our study is that the patients were already confirmed to be diabetics and eye exam was performed by trained 
ophthalmologists. Previous studies have established the vision threatening potential of diabetic retinopathy ${ }^{8}$. We found 29 eyes 15 patients to be presenting visual acuity at less than 6/60 level, however only two of these eyes were blind due to diabetic retinopathy. Cataract remained a major cause for blindness in these eyes, while the surgical removal of cataract may restore vision to these patients. There is potential for progression of diabetic retinopathy after cataract surgery, suggesting that these patients remain at an increased risk for blindness even if their cataract is removed ${ }^{9}$. We did not find any significant association between diabetic retinopathy and age, sex and hypertension. However we did not have sufficient cases of diabetic retinopathy to give us adequate statistical power to identify weak association. Consistent with other studies we found diabetic retinopathy to be strongly associated with duration of diabetes and poor glycemic control. The relatively low prevalence of diabetic retinopathy and blindness attributed to it may suggest that diabetic retinopathy requires less priority and attention than other major visually impairing diseases in India-like cataract and refractive errors that account for nearly $90 \%$ of current burden of blindness in India. However it has to be realised that the projected 57 million diabetics by 2025 in India may drastically alter the existing pattern of blindness in India. Improvement of healthcare system in India will probably translate into a large number of diabetics living longer, and thus more diabetics at risk for developing diabetic retinopathy. If diabetic retinopathy is not detected and treated early, the potential for vision loss is high, this will add to the burden of needless visual impairment and blindness in India. This will require improved networking between ophthalmologists and internists in India such that all diabetic patients receive a dilated fundus examination at regular intervals. Current treatment for diabetic retinopathies including laser are vision preserving at best, compared with the vision restoring potential for cataract surgeries or spectacles. The current cost for treating either cataract or refractive errors are higher than the costs involved for preventing or delaying onset of diabetic retinopathy. Tackling large burden of diabetic retinopathy will require a large number of trained personnel besides adequate infrastructure support. Training to treat vitreoretinal diseases including diabetic retinopathy is currently not part of all residency programmes in India. The number of ophthalmologists opting for post fellowship fellowships in vitreoretinal diseases is also not sizeable. The challenge this will pose to ophthalmic healthcare system in India is underscored when we consider that a cataract focussed national policy to prevent blindness in India has been able to increase annual cataract surgeries only to 3.5 million per year in 2000 after at least a decade of sustained effort.

There are only two ways to prevent blindness from diabetic retinopathy in patients with diabetes. One is to maintain strict glycemic control and second to do regular fundus examination of diabetic population.

\section{CONCLUSION}

Preventive measures have to be evolved to ensure that blindness due to diabetic retinopathy does not become a public health problem in India. Further studies are required to understand the risk factors for retinopathy and vision loss in this population.

\section{REFERENCES}

1. Klein R, Klein BE, Moss SE. visual impairment in diabetics. Ophthalmology 1984; 91: 1-9.

2. DwyerMS, Melton J, Ballard DJ, et al. Incidence of diabetic retinopathy and blindness, A population based study. Diabetes Care 1985; 8: 316-322.

3. The Eye Diseases Prevalence Research Group. The prevalence of diabetic retinopathy among adults in United States. Arch Ophthalmol 2004; 122: 552-563.

4. Wild S, Roglic G, Green A, Sicree R, King H. Global prevalence of diabetes estimated 
for the year 2000 and projection for 2030.

Diabetes Care 2004; 27: 1047-1053.

5. Mohan V, Ramachandran A, Snehalatha C.

High prevalence of maturity onset diabetes of young among Indians. Diabetes Care 1985; 8: 371-374.

6. Mohan V, Shanthirani CS, Deepa R. Glucose intolerance in a selected southern Indian population with special reference to family history, and life style. J Assoc physician India 2003; 51: 771-777.

7. King H, Aubert RE, Herman WH. Global burden of diabetes (1995-2025). Diabetes Care 1998; 21: 1414-31.

8. Aiello LM, Rand LI, Brones JC. Diabetic retinopathy in Joslin Clinic in patients with adult onset diabetes. Ophthalmology 1981; 88: 619-23.

9. Ruiz RS, Saatci OA. PCIOL implantation in eyes with active diabetic retinopathy. Am J Ophthalmol 1991; 111: 158-62. 